\title{
Numerical Study on Soil Collapse Using SPH with Elastic-Plastic Models
}

\author{
Dandan Dong1, Xuejun Zhou' ${ }^{1}{ }^{*}$ \\ 1 School of Mathematics and Physics, Huanggang Normal University, Huanggang, Hubei, China. \\ 2 School of Mechanics and Materials, Hohai University, Nanjing, Jiangsu, China \\ * Corresponding author. Tel.: +86-713-8621649; email: zhouxj@hhu.edu.cn \\ Manuscript submitted April 10, 2018; accepted July 23, 2018. \\ doi: 10.17706/ijapm.2018.8.4.66-78
}

\begin{abstract}
Numerical simulation of large deformation of soil based on constitutive models describing small deformation of soil in the framework of smoothed particle hydrodynamics (SPH) is investigated in this paper. In recent decades, the SPH method has been successfully used to simulate large deformation of soil. When SPH is used to simulate soil, constitutive models should be implemented in the SPH code in order to reflect the stress-strain relationship of soil. Nevertheless, typical soil constitutive models generally describe small deformation, and so the effects of them in the problem of simulating large deformation are worth researching. In this study, the SPH method with two elastic-plastic constitutive models i.e. Drucker-Prager model and the modified Cam-Clay model, are applied to simulate soil collapse. Numerical results indicate that there are superior differences in SPH simulation with the two constitutive models. Based on the obtained results, that constitutive models play an important role in simulation of large deformation of soil could be considered.
\end{abstract}

Key words: Elastic-plastic constitutive model, large deformation, smoothed particle hydrodynamics, soil collapse.

\section{Introduction}

Slope failures are complex natural phenomena that constitute a serious natural hazard, and impose heavy loss each year to human life and properties all over the world. Given this understanding, it is not surprising that landslides are rapidly becoming the focus of major scientific research, engineering study, and practices. To prevent or mitigate the landslide damage, one of research methodologies is numerical simulation technique, and it can understand and evaluate the processes that govern the behavior of the slopes economically and abundantly.

Slope failures belong to large deformation problems of soil, and dealing with such problems is generally difficult for finite element method (FEM) since FEM is suffered from grid distortions. Aim to simulate large deformation of soil, smoothed particle hydrodynamics (SPH) has been presented. SPH is a purely Lagrangian meshless method, compared with grid-based numerical methods, the SPH method can handle large deformation problems without mesh distortion.

SPH was originally developed for astrophysical applications by Lucy [1] and Monaghan \& Gingold [2]. Then it has been widely applied to dynamic response of material strength [3], [4], fluid flow [5], [6], etc. More recently, SPH application to soil mechanics was proposed by Maeda et al. [7], in which a simple non-linear elastic model was employed to simulate granular soil. SPH implemented by Drucker-Prager 
constitutive model was researched by Bui et al. [8], [9], which has demonstrated successful performance of SPH for simulating slope failure, landslide that are about large deformation in geotechnical engineering.

When SPH is utilized to mimic deformation of soil, the constitutive models should be implemented into the SPH code to describe the stress-strain relationship. Using different constitutive models, the soil is treated as an ideal material with different properties. In the problem of slope failures, the soil will experience the process of small deformation to large deformation, and exhibit the transition from a solid-like to a fluid-like behavior. The constitutive models of soils in SPH give rise to more realistic and accurate simulation results for large deformation problems [9].

Although there are many constitutive models of soil, frequently-used constitutive models are a few of elastic-plastic models, such as Drucker-Prager model (D-P model) and the modified Cam-Clay model (MCC model), because these models are relatively simple, easy programming and widespread application.

In this study, the two kinds of typical soil constitutive models are embedded into the SPH algorithm. The SPH method with D-P model and MCC model are adopted to simulate soil collapse. Based on the numerical and experimental results, the differences of the two constitutive models in simulation large deformation of soil are discussed.

The paper is organized as follows. In the next section the SPH method is briefly described. Then the SPH method implemented by soil constitutive models is presented. In the remaining section of the paper, the numerical framework is used in the simulations of soil collapse, and the results of numerical simulation are discussed and analyzed.

\section{Procedure for Paper Submission}

In the SPH method, the problem domain is discretized into a set of arbitrarily distributed particles, each particle representing a certain volume and mass of the material and carrying material information, such as velocity, acceleration, density, stress and so on. There are two key steps in SPH formulation. The first step is to represent a function and its derivatives as integral representation (termed as kernel approximation). The second step is particle approximation; it is done by replacing the integration in the integral representation of the field function and its derivatives with summations over all the corresponding values at the neighboring particles in a local domain.

In kernel approximation, the field function $f(\mathbf{x})$ at any point $\mathbf{x}$ is calculated according to an interpolation process over its neighboring particles that are within its influence domain $\Omega$ through

$$
f(\mathbf{x}) \approx \int_{\Omega} f\left(\mathbf{x}^{\prime}\right) W\left(\mathbf{x}-\mathbf{x}^{\prime}, h\right) d \mathbf{x}^{\prime}
$$

where $\mathbf{x}, \mathbf{x}^{\prime}$ are coordinate vectors contained in the influence domain $\Omega, d \mathbf{x}^{\prime}$ is infinitesimal volume element at point $\mathbf{x}^{\prime}, \mathrm{W}$ is kernel or smoothing function, $\mathrm{h}$ is the radius of the influence domain, depicting the influence of the smooth function.

The smooth function acts as a weight function in the approximation process, largely deciding the precision and stability of SPH method. The smooth function should satisfy the unity condition, the delta condition and the compact condition.

There are many kernel functions in the literature, such as bell-shaped function [1], Gaussian function [10], the cubic spline kernel function [11], the quartic spline function [12] etc. Among various smooth functions, the cubic spline kernel function is the most popular, and this kernel function is employed in the current study and can be expressed as 


$$
W\left(\mathbf{x}-\mathbf{x}^{\prime}, h\right)=W(R, h)=a_{d} \times \begin{cases}\frac{2}{3}-R^{2}+\frac{1}{2} R^{3}, & 0 \leq R<1 \\ \frac{1}{6}(2-R)^{3}, & 1 \leq R<2 \\ 0, & R \geq 2\end{cases}
$$

where $a_{d}$ is regularization factor, equals to $1 / h, 15 /\left(7 \pi h^{2}\right)$ and $3 / 2 \pi h^{3}$ for $1 \mathrm{D}, 2 \mathrm{D}$ and $3 \mathrm{D}$ conditions. $\mathrm{R}$ is the relative distance between two particles at points $\mathbf{x}$ and $\mathbf{x}^{\prime}$, and its expression is as follows

$$
R=\frac{\left|\mathbf{x}-\mathbf{x}^{\prime}\right|}{h}
$$

In particle approximation, the integral representation (1) concerning the SPH kernel approximation can be converted to discretized forms of summation over all the particles in the support domain. The particle approximation for a function at particle $i$ can finally be written as

$$
f\left(\mathbf{x}_{i}\right) \approx \sum_{j=1}^{N} \frac{m_{j}}{\rho_{j}} f\left(\mathbf{x}_{j}\right) W_{i j}
$$

where $m_{j}, \rho_{j}$ represent the mass and density of particle $j(=1,2, \ldots, \mathrm{N})$ respectively, in which $\mathrm{N}$ is the number of particles within the support domain of particle at point $\mathbf{x}$.

The particle approximation for the spatial derivative of the function $f(\mathbf{x})$ is

$$
\frac{\partial f\left(\mathbf{x}_{i}\right)}{\partial \mathbf{x}} \approx \sum_{j=1}^{N} \frac{m_{j}}{\rho_{j}} f\left(\mathbf{x}_{j}\right) \cdot \frac{\partial W_{i j}}{\partial \mathbf{x}_{i}}
$$

where

$$
W_{i j}=W\left(\mathbf{x}_{i}-\mathbf{x}_{j}, h\right)
$$

\section{Governing and Constitutive Equations of Soil}

\subsection{Governing Equations}

The governing equations for soil are obtained from the conservation equations of continuum mechanics, including the continuity and momentum equations expressed as [8]

$$
\begin{aligned}
\frac{D \rho}{D t} & =-\rho \frac{\partial v^{\alpha}}{\partial x^{\alpha}} \\
\frac{D v^{\alpha}}{D t} & =\frac{1}{\rho} \frac{\partial \sigma^{\alpha \beta}}{\partial x^{\beta}}+f^{\alpha}
\end{aligned}
$$

where $\alpha, \beta$ denote the Cartesian components $\mathrm{x}, \mathrm{y}$ and $\mathrm{z}$ with the Einstein convention applied to repeated indices; $\rho$ is the soil density; $v$ denotes the velocity; $f$ is gravity force; $D / D t$ represents the 
material derivative; $\sigma^{\alpha \beta}$ stands for the total tress tensor of soil, and it can be divided into an isotropic hydrostatic pressure $p$ and a deviatoric stress tensor $s^{\alpha \beta}$ :

$$
\sigma^{\alpha \beta}=-p \delta^{\alpha \beta}+s^{\alpha \beta}
$$

where $\delta^{\alpha \beta}$ is Kronecker's delta. In the framework of SPH, hydrostatic pressure $p$ is instead calculated directly from mean stress:

$$
p=-\frac{\sigma^{\gamma /}}{3}=-\frac{1}{3}\left(\sigma^{x x}+\sigma^{y y}+\sigma^{z z}\right)
$$

where $\sigma^{x x}, \sigma^{y y}$ and $\sigma^{z z}$ are the components of the stress tensor in the $\mathrm{x}, \mathrm{y}$ and $\mathrm{z}$ directions, and it can be defined that compressive stress corresponds to a negative stress.

According to the approximation method of SPH in the former section, the continuity and momentum Equations (7) and (8) can be approximated at particle $i$ as followed

$$
\begin{gathered}
\frac{D \rho_{i}}{D t}=\sum_{j=1}^{N} m_{j}\left(v_{i}^{\alpha}-v_{j}^{\alpha}\right) \frac{\partial W_{i j}}{\partial x_{i}^{\alpha}} \\
\frac{D v_{i}^{\alpha}}{D t}=\sum_{j=1}^{N} m_{j}\left(\frac{\sigma_{i}^{\alpha \beta}}{\rho_{i}^{2}}+\frac{\sigma_{j}^{\alpha \beta}}{\rho_{j}^{2}}\right) \frac{\partial W_{i j}}{\partial x_{i}^{\beta}}+f^{\alpha}
\end{gathered}
$$

It is noted that both of Equations (11) and (12) include the symmetrical structure, and it can reduce errors arising from the particle inconsistency problem [11].

In order to simulate large deformation of soil by SPH, the governing equations should be approximated using SPH interpolation functions. On the other hand, the soil constitutive models are important to describe the stress-strain relationship of soil in the SPH framework.

\subsection{Constitutive Equations of Soil}

In this subsection, we first give general formulations of elastic-plastic soil constitutive models, then obtain specific formulations of two kinds of typical soil constitutive models, i.e. D-P model and MCC model in the framework of SPH.

\subsubsection{General formulations}

The elastic deformation of soil is usually very small and does not represent an actual situation in geotechnical engineering problem, so the soil is regarded as a kind of plastic material has become a consensus. Within the framework of plasticity theory, the total strain rate tensor of soil $\dot{\varepsilon}^{\alpha \beta}$ is decomposed into two parts: an elastic strain rate tensor $\dot{\varepsilon}_{e}^{\alpha \beta}$ and a plastic strain rate tensor $\dot{\varepsilon}_{p}^{\alpha \beta}$ :

$$
\dot{\varepsilon}^{\alpha \beta}=\dot{\varepsilon}_{e}^{\alpha \beta}+\dot{\varepsilon}_{p}^{\alpha \beta}
$$

It is noted that the stress and strain are expressed by the form of rate because of the requirement of calculating in SPH method. 
The elastic strain rate tensor $\dot{\varepsilon}_{e}^{\alpha \beta}$ can be calculated by the generalized Hooke's law:

$$
\dot{\varepsilon}_{e}^{\alpha \beta}=\frac{\dot{s}^{\alpha \beta}}{2 G}+\frac{1-2 \nu}{3 E} \dot{\sigma}^{\gamma \gamma} \delta^{\alpha \beta}
$$

where $v$ is Poisson's ratio; E denotes Young's modulus; G represents the shear modulus.

The plastic strain rate tensor $\dot{\varepsilon}_{p}^{\alpha \beta}$ can be given by using the plastic flow rule:

$$
\dot{\varepsilon}_{p}^{\alpha \beta}=\dot{\lambda} \frac{\partial g}{\partial \sigma^{\alpha \beta}}
$$

where $\dot{\lambda}$ is the rate form of plastic multiplier, which can be computed from the consistency condition; $g$ is the plastic potential function.

The general stress-strain relationship for elastic-plastic soil can be derived as follows:

$$
\dot{\sigma}^{\alpha \beta}=2 G \dot{\varepsilon}^{\alpha \beta}+\left(K-\frac{2}{3} G\right) \dot{\varepsilon}^{\gamma /} \delta^{\alpha \beta}-\dot{\lambda}\left[\left(K-\frac{2}{3} G\right) \frac{\partial g}{\partial \sigma^{m n}} \delta^{m n} \delta^{\alpha \beta}+2 G \frac{\partial g}{\partial \sigma^{\alpha \beta}}\right]
$$

where $\dot{\varepsilon}^{\gamma /}=\dot{\varepsilon}^{x x}+\dot{\varepsilon}^{y y}+\dot{\varepsilon}^{z z}$ is the sum of the three normal strain components, $\mathrm{K}$ is the elastic bulk modulus.

\subsubsection{Drucker-Prager model}

As the failure surface of D-P model is smooth, this brings great convenience for numerical calculation and application. The D-P constitutive model with non-associated flow rule had been implemented in the SPH code in literature [9], and the soil is regarded as elastic-perfectly plastic material at that time. Herein, the process of implementation is just outlined.

The D-P yield criteria can be expressed as

$$
f\left(I_{1}, J_{2}\right)=\sqrt{J_{2}}+\alpha_{\phi} I_{1}-k_{c}
$$

where I 1 and $\mathrm{J} 2$ are the first and second stress invariants respectively, i.e.

$$
I_{1}=\sigma^{x x}+\sigma^{y y}+\sigma^{z z}, J_{2}=\frac{1}{2} s^{\alpha \beta} s^{\alpha \beta}
$$

$\alpha_{\phi}$ and $k_{c}$ are D-P constants, and in plane strain the two constants are computed by

$$
\alpha_{\phi}=\frac{\tan \phi}{\sqrt{9+12 \tan ^{2} \phi}}, k_{c}=\frac{3 c}{\sqrt{9+12 \tan ^{2} \phi}}
$$

where $\mathrm{c}$ and $\phi$ are cohesion and internal friction angle respectively.

For the non-associated plastic flow rule, the plastic potential function is written as 


$$
g=\sqrt{J_{2}}+3 I_{1} \sin \psi
$$

where $\psi$ denotes the dilatancy angle.

Substituting Equation (20) into Equation (16), and consideration of the contributions from rigid body rotation, the final stress-strain equation in SPH form for large deformation of the D-P model with non-associated flow rule can be expressed as

$$
\begin{aligned}
\frac{D \sigma_{i}^{\alpha \beta}}{D t} & =\sigma_{i}^{\alpha \gamma} \dot{\omega}_{i}^{\beta \gamma}+\sigma_{i}^{\imath \beta} \dot{\omega}_{i}^{\alpha \gamma}+2 G \dot{\varepsilon}_{i}^{\alpha \beta}+\left(K-\frac{2}{3} G\right) \dot{\varepsilon}_{i}^{\prime \prime} \delta_{i}^{\alpha \beta} \\
& -\dot{\lambda}_{i}\left(9 K \sin \psi \delta^{\alpha \beta}+\frac{G}{\sqrt{J_{2}}} s_{i}^{\alpha \beta}\right)
\end{aligned}
$$

with the following equation for the rate of change of plastic multiplier

$$
\dot{\lambda}_{i}=\frac{3 \alpha_{\phi} K \dot{\varepsilon}_{i}^{\prime \prime}+\left(G / \sqrt{J_{2}}\right) s_{i}^{\alpha \beta} \dot{\varepsilon}_{i}^{\alpha \beta}}{27 \alpha_{\phi} K \sin \psi+G}
$$

where $\dot{\omega}$ is spin rate tensor defined as

$$
\begin{aligned}
\dot{\omega}^{\alpha \beta} & =\frac{1}{2}\left(\frac{\partial v^{\alpha}}{\partial x^{\beta}}-\frac{\partial v^{\beta}}{\partial x^{\alpha}}\right) \\
& =\frac{1}{2}\left(\sum_{j=1}^{N} \frac{m_{j}}{\rho_{j}}\left(v_{j}^{\alpha}-v_{i}^{\alpha}\right) \frac{\partial W_{i j}}{\partial x_{i}^{\beta}}-\sum_{j=1}^{N} \frac{m_{j}}{\rho_{j}}\left(v_{j}^{\beta}-v_{i}^{\beta}\right) \frac{\partial W_{i j}}{\partial x_{i}^{\alpha}}\right)
\end{aligned}
$$

\subsubsection{The modified cam-clay model}

The MCC model is one of the most successful constitutive models for soil which originated from the work of Roscoe and his co-workers. The model is a critical state model, and the soil is considered as a hardening (softening) elastic-plastic material with a cap-shaped yield function

$$
f\left(p, q, p_{0}\right)=p^{2} M^{2}+q^{2}-M^{2} p p_{0}=0
$$

where $p$ defines as aforementioned, representing hydrostatic pressure; $\mathrm{q}$ is a state variable of deviatoric stress and can be defined as

$$
q=\sqrt{\frac{3}{2} s^{\alpha \beta} s^{\alpha \beta}}
$$

parameter M can be written by

$$
M=\frac{6 \sin \phi}{3-\sin \phi}
$$


$p_{0}$ is a hardening parameter concerned with plastic volumetric strain $\varepsilon_{p}^{\gamma /}$

$$
p_{0}=p_{a} \exp \left\{\frac{1+e_{0}}{\mu-\kappa} \varepsilon_{p}^{\gamma \gamma}\right\}
$$

where pa is the initial pressure; $\mu, \kappa$ denote compression and swelling index, respectively; $e_{0}$ is initial void ratio. The MCC model adopts associated flow rule, and plastic potential function $g$ is the same as the yield function $\mathrm{f}$. The final stress rate in SPH discretization form of MCC model can be expressed by

$$
\begin{aligned}
\frac{D \sigma_{i}^{\alpha \beta}}{D t} & =\sigma_{i}^{\alpha \gamma} \dot{\omega}_{i}^{\beta \gamma}+\sigma_{i}^{\gamma \beta} \dot{\omega}_{i}^{\alpha \gamma}+2 G \dot{\varepsilon}_{i}^{\alpha \beta}+\left(K-\frac{2}{3} G\right) \dot{\varepsilon}_{i}^{\gamma \gamma} \delta_{i}^{\alpha \beta} \\
& -\dot{\lambda}_{i}\left[K M^{2}\left(p_{0}-2 p_{i}\right) \delta^{\alpha \beta}+6 G s_{i}^{\alpha \beta}\right]
\end{aligned}
$$

with the following equation for the rate of change of plastic multiplier

$$
\dot{\lambda}_{i}=\frac{K M^{2}\left(p_{0}-2 p_{i}\right) \dot{\varepsilon}_{i}^{\gamma \gamma}+6 G \dot{\varepsilon}_{i}^{\alpha \beta} s_{i}^{\alpha \beta}}{K M^{4}\left(2 p_{i}-p_{0}\right)^{2}+12 G q_{i}^{2}+\frac{1+e_{0}}{\mu-\kappa} p_{i} p_{0} M^{4}\left(2 p_{i}-p_{0}\right)}
$$

\section{Algorithm Design}

\subsection{Stress Correction}

Since two kinds of elastic-plastic soil constitutive model were implanted in SPH method, the behavior of soil has to keep to the models i.e. the stress state must not lie outside the yield surface when plastic deformation takes place. However, the stress state of soil may leave the elastic domain during computational plasticity, which can lead to numerical errors. In order to avoid the errors, a stress correction algorithm is often used to numerically return the stress state to the yield surface.

\subsubsection{Stress correction on Drucker-Prager model}

For the stress correction on D-P model, the literature [9] has described in detail, here only the main idea is listed.

If the stress state of soil moves beyond the apex of the yield surface during computation, such as the stress state changes point A to point B illustrated in Figure 1, tension cracking will happen. The criterion of judging this situation is followed as

$$
-\alpha_{\phi} I_{1}^{n}+k_{c}<0
$$

which means the stress state of material at a time step n exceeds the apex of the yield surface. In this case, we shall keep the shearing stresses unchanged, and adjust the normal stress components to new values $\tilde{\sigma}$ so that the hydrostatic pressure corresponds to that at the apex (see Figure 1) followed as

$$
\tilde{\sigma}_{n}^{\alpha \beta}=\sigma_{n}^{\alpha \beta}-\frac{1}{3}\left(I_{1}^{n}-\frac{\alpha_{\phi}}{k_{c}}\right) \delta^{\alpha \beta}
$$




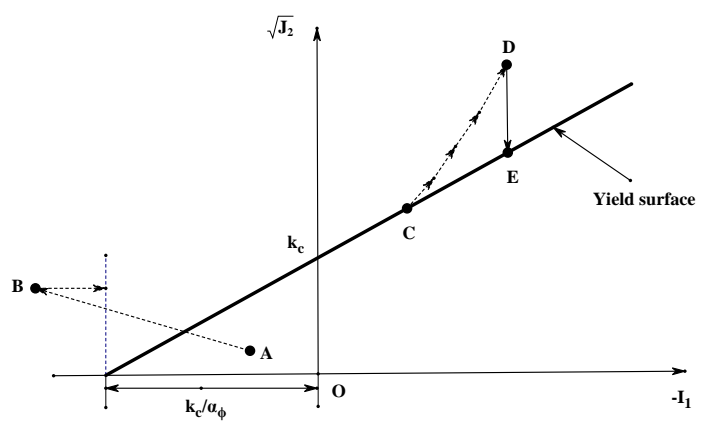

Fig. 1. Stress correction on Drucker-Prager model.

On the other hand, if a stress state lies far away from the yield surface, i.e. the path CD in Figure 1, the stress-rescaling procedure should be employed to return the stress state to the yield surface. The condition of this case can be written as

$$
r_{n}=\frac{-\alpha_{\phi} I_{1}^{n}+k_{c}}{\sqrt{J_{2}^{n}}}<1
$$

In such a circumstance, the deviatoric shear stress components are reduced in proportion to the scaling factor $r$, whereas the hydrostatic stress component I1 is left unchanged. The correction procedure can be expressed as

$$
\tilde{\sigma}^{\alpha \beta}=r^{n}\left(\sigma_{n}^{\alpha \beta}-\frac{1}{3} I_{1}^{n} \delta^{\alpha \beta}\right)+\frac{1}{3} I_{1}^{n} \delta^{\alpha \beta}
$$

Thus, the stress state will be pulled back from point $D$ to point $E$ on the yield surface (see Fig. 1).

\subsubsection{Stress correction on the modified cam-clay model}

When the soil is obedient to MCC model, the method of stress correction is quite different from D-P model, because MCC model has a cap-shaped yield function and a critical state line in stress space.

Similar to the second situation of D-P model, when a stress state exceeds the yield surface, such as the stress state at point $\mathrm{F}$ illustrated in Figure 2, which satisfies the following condition

$$
M^{2} p_{n}^{2}+q_{n}^{2}-M^{2} p_{0} p_{n}>0
$$

For the sake of returning the stress state $\mathrm{F}$ to the yield surface, we can make firstly a straight line between the stress point $\mathrm{F}$ and the center of the elliptic yield, and the intersection of the straight line and the yield surface is the correction stress point G (see Figure 2). The formula of correction procedure can be written as

$$
\beta_{n}^{o}=\frac{\left(2 p_{n}-p_{0}\right)^{2} p_{0} M^{2}}{M^{2}\left(2 p_{n}-p_{0}\right)^{2}+4 q_{n}^{2}}, \quad q_{n}=\frac{2 q_{n}\left(2 p_{n}-p_{0}\right) p_{0} M^{2}}{M^{2}\left(2 p_{n}-p_{0}\right)^{2}+4 q_{n}^{2}}
$$

It is noted that in the present study adopts the leapfrog (LF) algorithm for numerical integration, so the stress of soil is also interpolated at the half-time step $(n+1 / 2)$. 


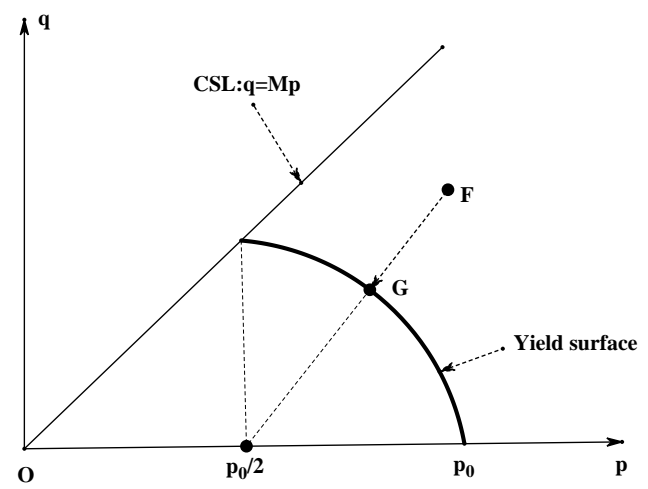

Fig. 2. Stress correction on the modified cam-clay model.

\subsection{Artificial Viscosity}

To improve the numerical stability and to damp out such undesirable oscillations, an additional artificial viscosity term $\Pi_{i j}$ proposed by Monaghan [10] is often added to the pressure term of the momentum Equation (8). Accordingly, Equation (8) is modified to,

$$
\frac{D v_{i}^{\alpha}}{D t}=\sum_{j=1}^{N} m_{j}\left(\frac{\sigma_{i}^{\alpha \beta}}{\rho_{i}^{2}}+\frac{\sigma_{j}^{\alpha \beta}}{\rho_{j}^{2}}-\Pi_{i j} \delta^{\alpha \beta}\right) \frac{\partial W_{i j}}{\partial x_{i}^{\beta}}+f^{\alpha}
$$

where

$$
\Pi_{i j}=\left\{\begin{array}{cc}
\frac{-\alpha_{\Pi} \bar{c}_{i j} \phi_{i j}+\beta_{\Pi} \phi_{i j}{ }^{2}}{\bar{\rho}_{i j}}, & \left(v_{i}-v_{j}\right) \cdot\left(x_{i}-x_{j}\right)<0 \\
0, & \left(v_{i}-v_{j}\right) \cdot\left(x_{i}-x_{j}\right) \geq 0
\end{array}\right.
$$

and where

$$
\phi_{i j}=\frac{h_{i j}\left(v_{i}-v_{j}\right) \cdot\left(x_{i}-x_{j}\right)}{\left|x_{i}-x_{j}\right|^{2}+\left(0.1 h_{i j}\right)^{2}}, \bar{c}_{i j}=\frac{1}{2}\left(c_{i}+c_{j}\right), \bar{\rho}_{i j}=\frac{1}{2}\left(\rho_{i}+\rho_{j}\right), h_{i j}=\frac{1}{2}\left(h_{i}+h_{j}\right)
$$

In the above equations, $\alpha_{\Pi}$ and $\beta_{\Pi}$ are constants that are all typically set around 1.0 [13]. $\mathrm{c}$ is the sound speed in soil, which typically lies in the range $450-600 \mathrm{~m} / \mathrm{s}$.

\subsection{Other Aspects}

When using the SPH method for soil, one numerical problem called tensile instability arises. The tensile instability is the situation that when particles are under tensile stress state, the motion of the particle becomes unstable. It could result in particle clumping or complete blowup in the computation. To deal with this problem, Bui et al. [8] have proposed to use an artificial stress method, originally invented by Gray et al. [14], and proved the effectiveness of this approach. SPH simulation may encounter the problem of particle deficiency near the solid boundary, and two types of boundary methods are widely used to resolve this problem: the ghost particles method [3] and the repulsive force method [4]. In the ghost particles method, layers of artificial particles are fixed on boundaries, carrying the same stress and density as the real SPH particles within the influence domain. Because the ghost boundary particles work well for straight 
boundaries, thus, this approach is adopted in the current study.

\section{Simulation Results and Discussions}

In this section, SPH with two constitutive models will be applied to simulate the gravitational flow following soil collapse, which involves an extremely large deformation and failure of soil. Through this study case, we will show that different constitutive models could present different behaviors of soil collapse at large deformation and post-failure. The simulation results of the SPH codes will be compared with Catherine and Trent's experiments [15], so physical properties of the materials in SPH simulations are the same to the experimental.

A total of 2501 real particles are used to form an initial rectangular soil area $4 \mathrm{~cm}$ in length and $6 \mathrm{~cm}$ in height with an initial smoothing length of $0.001 \mathrm{~m}$, and 1132 boundary particles are used to generate the solid boundaries. In the simulation, a column of soil is deposited between two walls, and the right wall is fixed whereas the left wall is withdrawn suddenly after the beginning of the simulation. The free-slip boundary condition is imposed to soil particles on the vertical wall while the no-slip boundary condition is applied to particles on the horizontal wall. The numerical model developed to reproduce the experimental setup is presented in Fig. 3. To visualize the internal dynamic of the flow, two different colors of otherwise identical layers of soil were used, and background grids with $2 \mathrm{~cm} \times 2 \mathrm{~cm}$ are bestowed.

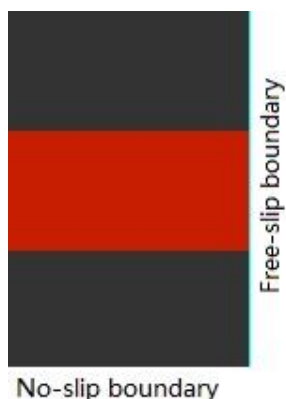

Fig. 3. Initial set up for the collapse test.

The material parameters used in the simulations are summarized in Table 1.

Table 1. Material Parameters

\begin{tabular}{|c|c|}
\hline Parameter & Value \\
\hline Young's modulus (MPa) & 1.0 \\
\hline Dilatancy angle (Degree) & 0 \\
\hline Internal friction angle $\left(^{\circ}\right)$ & 35 \\
\hline Cohesion $(\mathrm{Pa})$ & 0 \\
\hline Compression index & 0.085 \\
\hline Swelling index & 0.019 \\
\hline Initial void ratio & 0.68 \\
\hline Poisson's ratio & 0.3 \\
\hline Density $\left(\mathrm{g} \mathrm{cm}^{-3}\right)$ & 2.0 \\
\hline
\end{tabular}

Fig. 4 shows velocity nephograms of collapse in the simulations for SPH with D-P model and MCC model at representative times. The propagation of the shear bands from the crest to the toe of the columns can be clearly observed. The progressive failure process can be captured by the SPH code with the two models. In the SPH simulations, the thickness of shear band is mainly dominated by the smoothing length $h$. In addition, results of the SPH simulation with the two models are quite different. Soil simulated by D-P model acts more like granular material during the process of collapse, while soil looks more like clay in the SPH 
simulation of MCC model. As could be seen, soil in the D-P simulation performs much looser than in the MCC simulation, and soil collapses into blocks in some parts in the MCC simulation. [15] (top image), SPH with D-P model (middle image) and MCC model (bottom image)

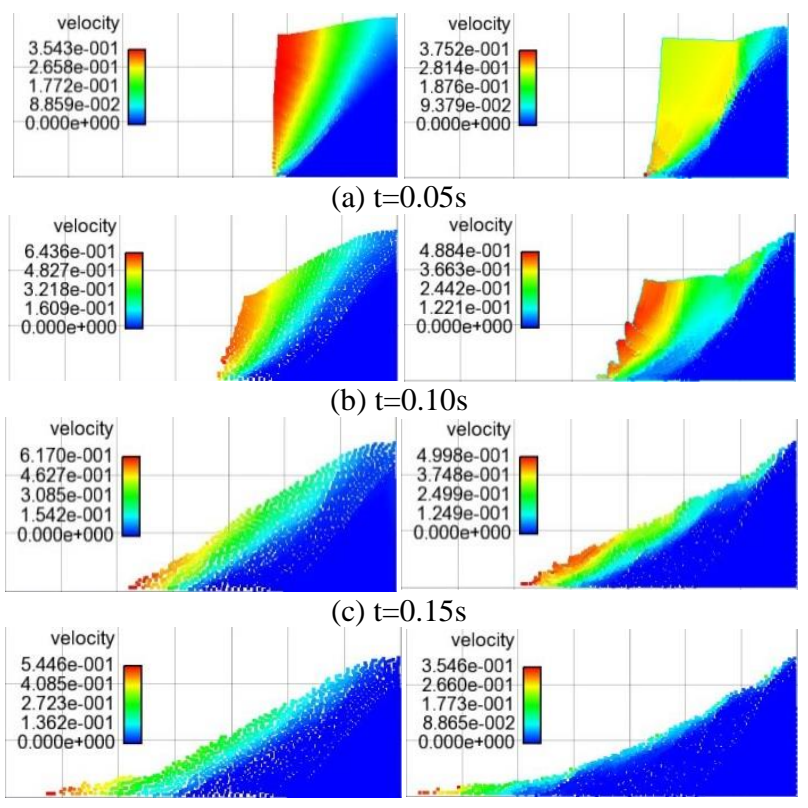

(d) $\mathrm{t}=0.20 \mathrm{~s}$

Fig. 4. Velocity nephograms of collapse in the simulations for SPH with D-P model (left images) and MCC model (right images) at representative times: (a) $t=0.05 \mathrm{~s}$; (b) $t=0.10 \mathrm{~s}$; (c) $t=0.15 \mathrm{~s}$; (d) $t=0.20 \mathrm{~s}$.

Final shapes of the deposit obtained by experimentally and numerically are given in Figure 5. Good agreement between simulations and experiment is found for the surface configurations; moreover, the surface of SPH simulation with MCC model is smoother and more analogous to experiment than D-P model. It can be found in MCC model that configuration of non-deformation sand on the right boundary is almost exactly alike as experiment. The repose angles of the two simulations are good agree with experiments, and these repose angles are less than the internal friction angle of $35^{\circ}$.

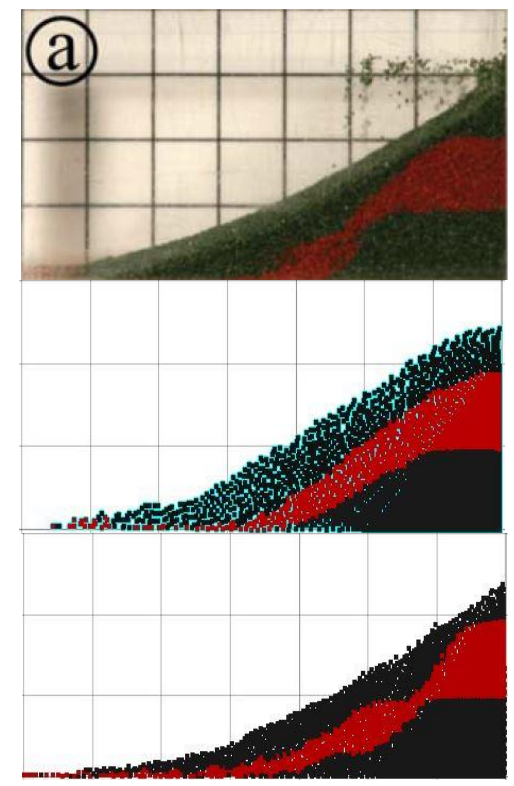

Fig. 5. Final shape of the deposit obtained by experiment. 
The different features of two constitutive models are possible reasons for aforementioned differences. Soil is deemed as elastic-perfectly-plastic material in simulation of D-P model; once the condition of failure is satisfied, the soil may flow, and it acts more like fluid. Whereas soil described by the modified Cam-Clay model takes on the property of gradual failure.

\section{Conclusions}

The SPH method embedded by two elastic-plastic constitutive models is applied to simulate large deformation of soil in the present study. The most popular elastic-plastic constitutive models i.e. Drucker-Prager model and the modified Cam-Clay model describe different stress-strain relationships of small deformation of soil. Constitutive models should be added to the governing equations when the SPH method is used to mimic the behaviors of soil. The proposed SPH codes with D-P model and MCC model are adopted to simulate soil collapse. The numerical results agree with the experimental well, but some differences are found in the SPH simulation with two models. For non-cohesive soil in our study, SPH with MCC model performs better than SPH with D-P model in term of surface configuration and shape of layers. Although constitutive models represent generally properties of soil during small deformation, they also play an important role in simulation of large deformation of soil.

In the future research, more constitutive models conforming to the characteristics of soil deformation may be embedded in the SPH method to find more and better details of large deformation of soil.

\section{Acknowledgment}

The research in this paper is funded by Huanggang Normal University Natural Science Foundation under Grant No. 201617603.

\section{References}

[1] Lucy, L. (1977). A numerical approach to testing the fission hypothesis. Astronomical Journal, 82, 1013-1024.

[2] Gingold, R. A., \& Monaghan, J. J. (1977). Smoothed particle hydrodynamics: theory and application to nonspherical stars. Monthly Notices of the Royal Astronomical Society, 181, 375-389.

[3] Libersky, L. D., Petschek, A. G., Carney, T. C., Hipp, J. R., \& Allahdadi, F. A. (1993). High strain Lagrangian hydrodynamics: a three dimensional SPH code for dynamic material response. Journal of Computational Physics, 109, 67-75.

[4] Benz, W., \& Asphaug, E. (1995). Simulations of brittle solids using smooth particle hydrodynamics. Computer Physics Communications, 87, 253-265.

[5] Monaghan, J. J. (1994). Simulating free surface flows with SPH. Journal of Computational Physics, 110, 399-406.

[6] Takeda. H., Miyama, S. M., \& Sekiya, M. (1994). Numerical simulation of viscous flow by smoothed particle hydrodynamics. Progress of Theoretical Physics, 92(5), 939.

[7] Maeda, K., \& Sakai, M. (2004). Development of seepage failure analysis procedure of granular ground with smoothed particle hydrodynamics (SPH) method. Journal of Applied Mechanics (JSCE), 7, 775-786.

[8] Bui, H. H. (2007). Lagrangian mesh-free particle method (SPH) for large deformation and post-failure of geomaterial using elasto-plastic constitutive models. Ph.D. Dissertation of Ritsumeikan University, Japan.

[9] Bui, H. H., Fukagawa, R., Sako, K., \& Ohno, S. (2008). Lagrangian meshfree particles method (SPH) for large deformation and failure flows of geomaterial using elastic-plastic soil constitutive model. Int J Numer Anal Meth Geomech, 32(12), 1537-1570. 
[10] Monaghan, J., J. (1992). Smoothed particle hydrodynamics. Annual Review of Astronomy and Astrophysics, 30, 543-574.

[11] Monaghan, J. J., \& Lattanzio, J. C. (1985). A refined particle method for astrophysical problems. Astronomic and Astrophysics, 149, 135.

[12] Morris, J., P. (1996). Analysis of SPH with applications. Ph.D. Thesis, Mathematics Department, Monash University, Melbourne, Australia.

[13] Monaghan, J., J. (1988). An introduction to SPH. Computer Physics Communication, 48, 89-96.

[14] Gray, J. P., Monaghan, J. J., \& Swift, R., P. (2001). SPH elastic dynamics. Computer Methods in Applied Mechanics and Engineering, 190, 6641-6662.

[15] Catherine, M., \& Trent, T. (2008). Scaling the final deposits of dry cohesive granular columns after collapse and quasi-static fall. Physics of Fluids, 20(3).

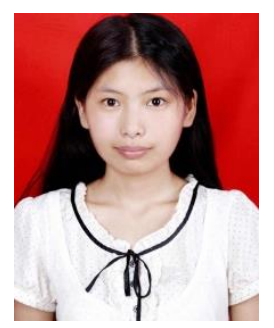

Dandan Dong was born in Henan, China, in 1997. Now, she is a B.S. candidate in mathematics and applied mathematics from Huanggang Normal University, China since 2016.

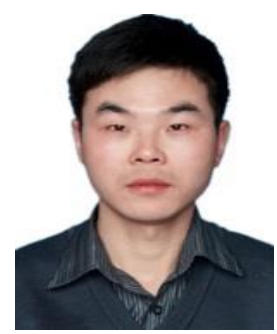

Xuejun Zhou was born in Hubei, China, in 1981. He received the B.S. in mathematics and applied mathematics from Hubei Engineering University, China, in 2003, and the M.S. in applied mathematics from Hubei University, China, in 2006. Now, he is a Ph.D. candidate in solid mechanics from Hohai University, China, since 2014.

In 2006, he joined the college of mathematics and physics, Huanggang Normal University, China, as a tutor, and in 2009 became a lecturer. Since December 2017, he has been with the college of mathematics and physics, Huanggang Normal University, China, where he was an associated professor. His current research interests include numerical study on large deformation of geomaterials, and assessment of slope stability.

Mr. Zhou was a membership of the college of mathematics and physics, Huanggang Normal University, China, for life. 